\title{
Micro meets macro
}

\begin{abstract}
A microscopic one-photon subsystem can be entangled with a macroscopic subsystem of thousands of photons: such hybrid micro-macro entanglement, now efficiently produced and verified, should be useful for quantum metrology and for fundamental tests of quantum mechanics.
\end{abstract}

\section{Fabio Sciarrino}

$\mathrm{H}$ ow macroscopic can a quantum state be and still retain its entanglement with a companion microscopic system? In recent years considerable experimental effort has been devoted to the observation of micro-macro entangled states. Writing in Nature Physics, Natalia Bruno et al. ${ }^{1}$ and Alexander Lvovsky et al. ${ }^{2}$ describe two remarkable experiments that report micromacro optical entanglement, with the macro subsystem involving as many as $10^{8}$ photons.

One of the fundamental questions of quantum physics is where the boundary between the classical and quantum worlds lies. Whereas microscopic systems obey the quantum superposition principle, the macroscopic realm is characterized by mutually exclusive realities. Is it then possible to establish a bridge between these two regimes? Such a situation is realised in Erwin Schrödinger's paradigmatic thoughtexperiment in which a cat (as an example of a macroscopic system) is locked in a box with a decaying atom (the microscopic counterpart). The quest for micro-macro entanglement reflects the recent growing interest in observing quantum phenomena in larger physical systems - quantum superpositions of optical fields in a cavity, trapped ions, fullerene molecules, ultracold atoms and superconducting circuits.

A direct approach to the generation of micro-macro entanglement is to start from a micro-micro quantum state and then amplify one of the two subsystems. This quantum amplification of entanglement was pioneered ${ }^{3}$ in 1998 and was later pursued in various experiments ${ }^{4}$. However, the amplification process is not a trivial operation in the quantum domain, where the no-cloning theorem implies that the amplified field is not a simple copy of the initial micro system ${ }^{3}$. By exploiting parametric amplification from a nonlinear crystal, micro-macro systems have been experimentally generated, but the demonstration of entanglement was not conclusive $e^{4-6}$. In fact, this would require the measurement of the macroscopic part of the superposition with single-photon resolution $^{5-7}$, or adopting high-efficiency

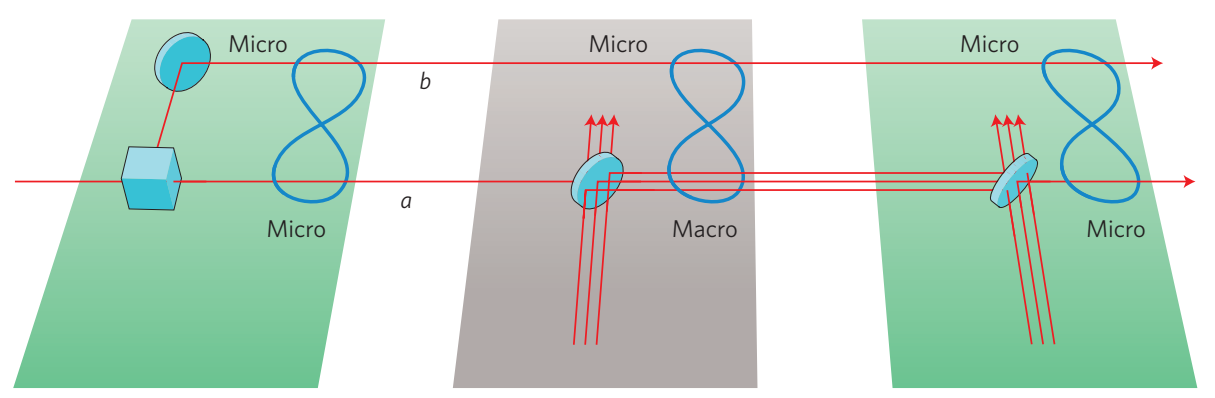

Figure 1 | Experimental set-up for the observation of micro-macro entanglement. A micro-micro entangled state is generated by sending a single photon into a beamsplitter. The micro subsystem $a$ is combined with a strong light beam on an unbalanced beamsplitter to transfer the state to the macro regime. Finally the macro part is displaced back to the (micro) single-photon level to verify the presence of entanglement.

homodyne measurements ${ }^{7,3}$ - and both scenarios are technically very challenging.

Along the lines of a recent proposal ${ }^{8}$, Bruno et al. ${ }^{1}$ and Lvovsky et al. ${ }^{2}$ designed and implemented a clever approach that exploits linear optics instead of nonlinear amplification, leading to reliable state generation and measurement. The two experiments exploit the concept of entanglement between one photon and the vacuum 9 : a single photon is sent into a beamsplitter, the output state being the coherent superposition of a state where the optical mode $a$ is in vacuum and mode $b$ carries one photon, and the complementary case, where $a$ is populated (Fig. 1). This embodies the initial micro-micro entangled state. To take the quantum micro system $a$ all the way to the macroscopic regime, both experiments adopt a smart trick: mode $a$ is allowed to interfere with an intense light beam on a highly unbalanced beamsplitter ${ }^{1,2}$. This process - which effectively displaces the state of mode $a$ in its phase space - is described by a local unitary transformation, thus fully preserving the entanglement of the initial state. However, now the overall state involves a micro-macro system.

Several questions pop up: how do we detect this hybrid entanglement? Does the sensitivity of the micro-macro state to the environmental coupling increase with the size of the macro system? Is this a 'proper' micro-macro entangled state?

To verify the entanglement of the final state the macro-system was displaced back to the single photon level (by another local unitary analogous to the first displacement operation). There the existence of micromicro quantum correlations provides evidence for the existence of entanglement in the micro-macro state.

The experiments may seem 'easy' to perform, but the larger the size of the macroscopic optical field, the higher the sensitivity to phase fluctuations. This is the behaviour one would expect from a macroscopic system with quantum features. To achieve a highly phase-stable set-up, Bruno et al. ${ }^{1}$ built a proper stabilization system, whereas in order to avoid instabilities Lvovsky et al. ${ }^{2}$ exploited beams with orthogonal polarization propagating along the same optical mode.

The final question relates to the effective 'size' of the quantum macroscopicity does the macro field corresponding to the displacement of zero or one photon essentially behave like a cat which can be either alive or dead, as in Schrödinger's original paradox? To this end, different criteria have been proposed and the most suitable one is still debated within the community ${ }^{10}$. 
The increasing sensitivity of the system to environmental decoherence can be used to support the macroscopic character of the quantum state. However, to be even more faithful to Schrödinger's paradigm, one needs to generate two macroscopic states that can be classically discriminated. This condition can be translated into the single-shot state distinguishability using detectors with no microscopic resolution. This is fulfilled for the implemented micro-macro states ${ }^{1,2}$, albeit with some intrinsic error probability in distinguishing between the two states. Whereas the discrimination efficiency is predicted to be $90 \%$ (ref. 2), due to technical imperfections Lvovsky et al. achieved a discrimination efficiency of about $68 \%$.

The two experiments will offer new perspectives and will certainly inspire the design of other physical platforms for similar studies. A relevant challenge is to conceive a viable method for the direct measurement of micro-macro entanglement without displacing the macroscopic quantum state back to the single-photon level. Other intriguing directions may involve the coupling of optical fields to atomic or mechanical systems through resonant reflection or radiation pressure mechanisms to transfer the superposition of the optical fields to a superposition of massive objects. Finally, one could apply the displacement operations on both subsystems to obtain a macro-macro entanglement.

\author{
00185 Roma, Italy. \\ e-mail:fabio.sciarrino@uniroma1.it \\ References \\ 1. Bruno, N. et al. Nature Phys. 9, 545-548 (2013). \\ 2. Lvovsky, A. et al. Nature Phys. 9, 541-544 (2013). \\ 3. De Martini, F. \& Sciarrino, F. Rev. Mod. Phys. \\ 84, 1765 (2012). \\ 4. De Martini, F., Sciarrino, F. \& Vitelli, C. Phys. Rev. Lett. \\ 100, 253601 (2008). \\ 5. Sekatski, P., Brunner, N., Branciard, C., Gisin, N. \& Simon, C. \\ Phys. Rev. Lett. 103, 113601 (2009). \\ 6. Spagnolo, N., Vitelli, C., Sciarrino, F. \& De Martini, F. Phys. Rev. A \\ 82, 052101 (2010) \\ 7. Spagnolo, N., Vitelli, C., Paternostro, M., De Martini, F. \& \\ Sciarrino, F. Phys. Rev. A 84, 032102 (2011). \\ 8. Sekatski, P. et al. Phys. Rev. A 86, 060301 (2012). \\ 9. Lombardi. E. Sciarrino, F., Popescu, S. \& De Martini, F. \\ Phys. Rev. Lett. 88, 070402 (2002). \\ 10. Korsbakken, J. I., Wilhelm, F. K. \& Whaley, K. B. Europhys. Lett. \\ 89, 30003 (2010).
}

Published online: 21 July 2013

\section{QUANTUM POINT CONTACTS}

\section{Double or nothing?}

The role that quasi-bound spins play in the ' 0.7 anomaly' is controversial. One study suggests that two or more quasi-bound spins may be involved; another advocates that the 0.7 anomaly is a density-of-states effect, needing neither a quasi-bound spin nor spontaneous spin polarization.

\section{Adam Micolich}

am sometimes approached by

undergraduate students bemoaning their

first 'modern' physics course: "It's so 1940s, how are one-dimensional potential-well problems still relevant today?" My answer is the quantum point contact (QPC) - a beautiful example of the one-dimensional quantum oscillator in a modern context, and a nanoscale electronic device that is still subject to active research.

The QPC is a short, narrow, $1 \mathrm{D}$ aperture separating two $2 \mathrm{D}$ electron reservoirs in an $\mathrm{AlGaAs} / \mathrm{GaAs}$ heterostructure (Fig. 1a). The QPC is usually defined electrostatically; its width controlled by the voltage applied to nanoscale 'gate' electrodes. The classic signature of the QPC is a step-like plot of the electrical conductance versus gate voltage, with plateaux at integer multiples of $2 e^{2} / h$ ( $e$ is electron charge, $h$ is Planck's constant). These plateaux are easily explained, but the origin of the anomalous plateau ${ }^{1,2}$ that is consistently observed at $0.7 \times 2 e^{2} / h$ is still an enigma.

Two recent papers in Nature offer new insight into this 0.7 anomaly. Florian Bauer and colleagues present a 1D model where an interaction-modified $1 \mathrm{D}$ density-of-states (DOS) produces the 0.7 anomaly without needing spin-polarization or quasi-bound states $^{3}$. Muhammad Javaid Iqbal and co-workers show interesting new data linking the 0.7 anomaly to one or more spontaneously localized states (SLSs) formed along the QPC ${ }^{4}$.

At its simplest, the QPC is a saddle-point potential hosting discrete levels $E_{\mathrm{n}}$ called '1D subbands' (Fig. 1b). More negative gate voltage acts at the centre of the QPC to raise the barrier along $x$ and narrow the well along $y$, driving the 1D subbands upwards in energy. Conduction in each $1 \mathrm{D}$ subband ceases as it crosses the Fermi energy of the adjacent $2 \mathrm{D}$ reservoirs. The $1 \mathrm{D}$ DOS is proportional to $\left(E-E_{n}\right)^{-1 / 2}$, fortuitously cancelling the electron velocity, which depends on $E^{1 / 2}$. This gives the conductance steps their clean shape and equal height.

Bauer et al. ${ }^{3}$ use a $1 \mathrm{D}$ tight-binding model to show that the barrier at the QPC centre and electron-electron interactions combine to modify the $1 \mathrm{D}$ local DOS (LDOS) at the centre of the QPC. The saddle-point barrier slows the traversing electrons considerably, with two effects. First, it converts the $\left(E-E_{1}\right)^{-1 / 2}$ form of the 1D DOS into a ridgelike feature (Fig. 1c) - which Bauer et al. call the 'Hove ridge' - giving maximal LDOS above, rather than at, $E_{1}$. Second, it strongly enhances electron-electron interactions at the QPC centre, as proposed in ref. 5. The interactions and LDOS act somewhat iteratively: interactions are enhanced where the LDOS is high, which further modifies the LDOS (particularly near the Hove ridge). The net result is a strong modification of the conductance step between 0.5 and $0.9 \times 2 e^{2} / h$. Screening limits this interaction effect to only the lowest $1 \mathrm{D}$ subband at the QPC centre ${ }^{5}$. Most of the experimentally observed behaviours of the 0.7 anomaly are reproduced by the $1 \mathrm{D}$ model developed by Bauer and colleagues ${ }^{3}$.

Another proposed origin for the 0.7 anomaly is Kondo physics ${ }^{6}$. The parallels between the 0.7 anomaly in QPCs and the quantum-dot Kondo effect were first highlighted by Cronenwett and colleagues ${ }^{7}$, who focused on an anomalous peak in the differential conductance versus the d.c. source-drain bias voltage, known as the zero-bias anomaly (ZBA). A key contention with a Kondo scenario is that a QPC is open, and should thus not support a closed, dotlike state ${ }^{2}$.

It has since been shown using spindensity functional theory that Friedel oscillations may generate a single quasibound spin at the QPC centre ${ }^{8}$ (Fig. 1d). The observation of Fano resonances in devices that feature pairs of nearby QPCs 